\title{
A one-parameter Family of Transforms, Linearizing Convolution Laws for Probability Distributions
}

\author{
Alexandru Nica \\ Department of Mathematics, University of California, Berkeley, California 94720, U.S.A \\ (E-mail: nica@cory.berkeley.edu)
}

Received: 21 March 1994

\begin{abstract}
We study a family of transforms, depending on a parameter $q \in[0,1]$, which interpolate (in an algebraic framework) between a relative (namely: $\left.-i z(\log \mathscr{F}(\cdot))^{\prime}(-i z)\right)$ of the logarithm of the Fourier transform for probability distributions, and its free analogue constructed by D. Voiculescu ([16, 17]). The classical case corresponds to $q=1$, and the free one to $q=0$.

We describe these interpolated transforms: (a) in terms of partitions of finite sets, and their crossings; (b) in terms of weighted shifts; (c) by a matrix equation related to the method of Stieltjes for expanding continued $J$-fractions as power series. The main result of the paper is that all these descriptions, which extend basic approaches used for $q=0$ and/or $q=1$, remain equivalent for arbitrary $q \in[0,1]$.

We discuss a couple of basic properties of the convolution laws (for probability distributions) which are linearized by the considered family of transforms (these convolution laws interpolate between the usual convolution - at $q=1$, and the free convolution introduced by Voiculescu - at $q=0$ ). In particular, we note that description (c) mentioned in the preceding paragraph gives an insight of why the central limit law for the interpolated convolution has to do with the $q$-continuous Hermite orthogonal polynomials.
\end{abstract}

\section{Introduction and Statement of Results}

We will work with probability distributions having finite moments of all orders, and we will consider a simplified algebraic approach, where the sequence of moments $\left(\int_{\mathbf{R}} t^{n} d \mu(t)\right)_{n=0}^{\infty}$ is addressed, rather than the distribution $\mu$ itself. Thus we will think (in this simplified approach) of distributions as of linear functionals on the algebra $\mathbf{C}\langle X\rangle$ of polynomials in an indeterminate, and write " $\mu(f)$ " instead of " $\int f d \mu$ ", for $f$ a polynomial. We denote the space of such objects by $\Sigma$; i.e.,

$$
\sum=\{\mu: \mathbf{C}\langle X\rangle \rightarrow \mathbf{C} \mid \mu \text { linear }, \mu(1)=1\} .
$$

For $\mu_{1}, \mu_{2} \in \Sigma$, their convolution product (which is denoted in this paper by $\mu_{1} \cdot \mu_{2}$ because the symbol " $\star$ " is used for free products) gets the expression:

$$
\left(\mu_{1} \cdot \mu_{2}\right)(f)=\left(\mu_{1} \otimes \mu_{2}\right)\left(f\left(X_{1}+X_{2}\right)\right), \quad f \in \mathbf{C}\langle X\rangle ;
$$


the tensor product $\mu_{1} \otimes \mu_{2}$ in (1.2) is viewed as the linear functional on the algebra of polynomials in $X_{1}$ and $X_{2}$, which has $\left(\mu_{1} \otimes \mu_{2}\right)\left(X_{1}^{m} X_{2}^{n}\right)=\mu_{1}\left(X_{1}^{m}\right) \mu_{2}\left(X_{2}^{n}\right), m, n \geqq 0$.

In the work of D. Voiculescu $([16,17])$, a theory of free convolution of distributions was developed, which parallels the usual convolution theory, in a context where tensor products are replaced by free products. More precisely, the free convolution of $\mu_{1}, \mu_{2} \in \Sigma$, denoted by $\mu_{1} \boxplus \mu_{2}$, is described in the same way as in (1.2), but where the tensor product of $\mu_{1}$ and $\mu_{2}$ is replaced by their free product $\mu_{1} \star \mu_{2}$ :

$$
\left(\mu_{1} \boxplus \mu_{2}\right)(f)=\left(\mu_{1} \star \mu_{2}\right)\left(f\left(X_{1}+X_{2}\right)\right), \quad f \in \mathbf{C}\langle X\rangle .
$$

For the definition of $\mu_{1} \star \mu_{2}$, which is a linear functional on the algebra of noncommutative polynomials in $X_{1}$ and $X_{2}$, see for instance Sect. 1.5 of [18].

Our emphasis will be on transforms which linearize convolution. The existence of such transforms is an important point in the theory of both usual and free convolution. On one hand, as it is well-known, usual convolution is linearized by the logarithm of the Fourier transform: $\log \mathscr{F}\left(\mu_{1} \cdot \mu_{2}\right)=\log \mathscr{F}\left(\mu_{1}\right)+\log \mathscr{F}\left(\mu_{2}\right), \mu_{1}, \mu_{2} \in$ $\Sigma$. In the present context, the Fourier transform of a distribution $\mu$ is viewed as a formal power series, $\left((\mathscr{F}(\mu))(z)=\sum_{n=0}^{\infty} \frac{t^{n} \mu\left(X^{n}\right)}{n !} z^{n}\right.$, and the logarithm of a formal power series $\varphi$ with $\varphi(0)=1$ is taken as $\log \varphi=\sum_{n=1}^{\infty} \frac{1}{n}(-1)^{n+1}(\varphi-1)^{n}$; hence for $\mu \in \Sigma, \log \mathscr{F}(\mu)$ is viewed here as a formal power series vanishing at zero. On the other hand, free convolution is linearized by a certain $R$-transform, defined in $[16,17]$ via a construction involving "formal" Toeplitz operators. For $\mu \in \Sigma, R(\mu)$ is also a formal power series vanishing at zero, and we have the formula $R\left(\mu_{1} \boxplus \mu_{2}\right)=R\left(\mu_{1}\right)+R\left(\mu_{2}\right), \mu_{1}, \mu_{2} \in \Sigma$.

It is easily seen that both the logarithm of the Fourier transform and the $R$ transform are bijections between the space of distributions $\Sigma$ of (1.1), and the space

$$
\Theta=\left\{\sum_{n=1}^{\infty} \alpha_{n} z^{n} \mid \alpha_{1}, \alpha_{2}, \alpha_{3}, \ldots \in \mathbf{C}\right\}
$$

of formal power series vanishing at zero. Therefore one can in fact define both the usual and free convolution as the operations on $\Sigma$ obtained by transporting from $\Theta$ the pointwise addition, via the appropriate transforms.

In this paper we point out a remarkable family of bijective transforms $R_{q}$ : $\Sigma \rightarrow \Theta$, depending on a parameter $q \in[0,1]$, which interpolate between the $R$ transform (at $q=0)$, and a relative - namely " $-i z(\log \mathscr{F}(\cdot))^{\prime}(-i z)$ "- of the logarithm of the Fourier transform (at $q=1$ ). The $R_{q}$ 's bring along a family of convolution laws $\boxplus_{q}, q \in[0,1]$, on the space $\Sigma$ of distributions, which interpolate between free convolution (at $q=0$ ) and usual convolution (at $q=1$ ); more precisely, $\boxplus_{q}$ is the operation on $\Sigma$ obtained by transporting from $\Theta$ the pointwise addition, via the $R_{q}$-transform.

The main goal of the paper is to show that the $R_{q}$-transforms $(0 \leqq q \leqq 1)$ can be described in several ways, corresponding to some basic approaches used for $q=0,1$. The point consists, of course, in proving the non-trivial fact that all these descriptions remain equivalent for arbitrary $q \in[0,1]$.

We have started our work from the approach of Voiculescu, in the case $q=0$, where the $R$-transform is defined in terms of formal Toeplitz operators, i.e. in terms of certain generating functions having as variable the unilateral shift on a separable Hilbert space. The $R_{q}$-transform is obtained from this by putting on the shift the weight (depending on the parameter $q$ ) which was considered in connection to the 
" $q$-commutation relations," a remarkable interpolation between the canonical commutation and the canonical anti-commutation relations - see [3]. (The same weighted shift also appears in the context of the quantum $S U(2)$ group - see for instance [20]). The $q$-commutation relations seem to be well-suited to "quantum probabilistic" considerations (see e.g. [12]), and the idea of defining the $R_{q}$-transform in this way comes naturally on this line of thought.

On the other hand, it turns out that the $n^{\text {th }}$ moment of a distribution $\mu$ is expressed in terms of the coefficients of its $R_{q}$-transform via a summation formula over the set of partitions of $\{1, \ldots, n\}$; in the case $q=1$, this is equivalent to the well-known formula relating the moments of $\mu$ and its so-called "cumulants" (see for instance [11], Sect. II.12.8). We mention that the existence of such a formula had also been noticed in the case $q=0$; more precisely, it had been shown by R. Speicher [13] that in this case one has to consider in the summation formula only those partitions of $\{1, \ldots, n\}$ which are non-crossing (in the sense of [8]). The interpolation (in the summation formula for the moments) between the set of non-crossing partitions and the set of all partitions turns out to be due to a factor " $q^{c_{o}(\pi) "}$ in the general term of the sum, where $c_{o}(\pi)$ is a number accounting in some sense for "how many crossing has the partition $\pi$ " (see Notation 1.1.A below for the exact definition).

Moreover, it turns out to be possible to give a third characterization of the $R_{q^{-}}$ transforms, in terms of a matrix equation related to the one involved in the method of Stieltjes for expanding continued $J$-fractions as power series. This characterization gives an insight of why in a certain (important) particular case, taking the $R_{q}$ transform has to do with the $q$-continuous Hermite orthogonal polynomials (see 1.6, 4.2 below).

In all the approaches we consider, the inverse of the $R_{q}$-transform, $R_{q}^{-1}: \Theta \rightarrow \Sigma$ is actually defined first, and then $R_{q}$ is defined as $\left(R_{q}^{-1}\right)^{-1}$. We mention that all the considerations of the paper are actually valid for $q \in(-1, \infty)$; and moreover, what happens at $q=-1$ is that $R_{q}^{-1}$, which can still be defined, is no longer bijective.

In order to state explicitly the main result of the paper (Theorem 1.2 below), we need to set a few notations.

\subsection{Notations}

A. Partitions. For $n \geqq 1$, we denote by $\mathscr{P}(\{1, \ldots, n\})$ the set of partitions of $\{1, \ldots, n\}$. For $\pi \in \mathscr{P}(\{1, \ldots, n\})$ and $1 \leqq m_{1}, m_{2} \leqq n$, we will write " $m_{1} \stackrel{\pi}{\sim} m_{2}$ " for the fact that $m_{1}$ and $m_{2}$ are in the same class (block) of $\pi$.

Recall that a partition $\pi$ of $\{1, \ldots, n\}$ is said to be non-crossing (notion introduced in [8]) if there is no 4-tuple $\left(m_{1}, m_{2}, m_{3}, m_{4}\right)$ such that $1 \leqq m_{1}<m_{2}<$ $m_{3}<m_{4} \leqq n, m_{1} \stackrel{\pi}{\sim} m_{3} \stackrel{\pi}{\sim} m_{2} \stackrel{\pi}{\sim} m_{4}$.

We will call left-reduced number of crossings of $\pi \in \mathscr{P}(\{1, \ldots, n\})$ the number:

$$
c_{o}(\pi)=\operatorname{card}\left\{\begin{array}{ll}
\left(m_{1}, m_{2}, m_{3}, m_{4}\right) \quad \begin{array}{l}
\mid 1 \leqq m_{1}<m_{2}<m_{3}<m_{4} \leqq n, m_{1} \stackrel{\pi}{\sim} m_{3} \\
\mid m_{2} \sim m_{4}, \text { each of } m_{1}, m_{2} \text { is minimal } \\
\text { in the class of } \pi \text { containing it }
\end{array}
\end{array}\right\} .
$$

The words "left-reduced" in the name of $c_{o}(\pi)$ refer to the fact that rather than counting all the 4-tuples mentioned in the preceding paragraph, we impose a more 
restrictive condition "on the left" (the minimality requirement on $m_{1}$ and $m_{2}$ implies of course $\left.m_{1} \stackrel{\pi}{\sim} m_{2}\right)$. It is easy to check, however, that a partition $\pi \in \mathscr{P}(\{1, \ldots, n\})$ is non-crossing if and only if it has $c_{o}(\pi)=0$.

B. Matrices. We will consider infinite complex matrices of the form $M=\left(x_{l, j}\right)_{i, j \geqq 0}$. The $(i, j)$-entry of a matrix $M$ will be denoted by $(M)_{i, j}$. We will call diagonal height of $M$ the number

$$
d h(M)=\sup \left\{j-i \mid i, j \in \mathbf{N},(M)_{i j} \neq 0\right\} \subseteq \mathbf{Z} \cup\{-\infty, \infty\},
$$

where " $d h(M)=-\infty$ " is, by convention, equivalent to " $M=0$ ".

We denote by $\mathscr{M}$ the set of infinite matrices $M$ having $d h(M)<\infty$. Then $\mathscr{M}$ is a unital algebra, with the usual operations with matrices. Note that $d h(M+N) \leqq$ $\max (d h(M), d h(N))$, and that $d h(M N) \leqq d h(M)+d h(N)$ for $M, N \in \mathscr{M}$.

We denote by $\omega$ the linear functional $\mathscr{M} \rightarrow \mathbf{C}$ defined by

$$
\omega(M)=(M)_{0,0}, \quad M \in \mathscr{M} .
$$

For $M \in \mathscr{M}$ we denote by $1 \oplus M$ the matrix with entries

$$
(1 \oplus M)_{i, j}= \begin{cases}(M)_{i-1, j-1}, & \text { if } i, j \geqq 1 \\ 1, & \text { if } i=j=0 \\ 0, & \text { otherwise }\end{cases}
$$

(this is of course in $\mathscr{M}$, with $d h(1 \oplus M)=\max (d h(M), 0)$ ).

C. Weighted shifts. We will consider the following remarkable family of weighted shifts on $l^{2}(\mathbf{N})$ (first studied by S.L. Woronowicz [20]): for $0 \leqq q<1$, denote by $S_{q}$ the operator on $l^{2}(\mathbf{N})$ determined by $S_{q} \delta_{n}=\sqrt{[n+1]_{q}} \delta_{n+1}, n \geqq 0$, where $\left(\delta_{n}\right)_{n=0}^{\infty}$ is the canonical basis of $l^{2}(\mathbf{N})$, and where we use the customary notation $[m]_{q}=1+q+\cdots+q^{m-1}, m \geqq 1$. The adjoint $S_{q}^{*}$ of $S_{q}$ is determined by $S_{q}^{*} \delta_{0}=0, S_{q}^{*} \delta_{n}=\sqrt{[n]_{q}} \delta_{n-1}, n \geqq 1 . S_{q}$ and $S_{q}^{*}$ satisfy the important relation $S_{q}^{*} S_{q}=q S_{q} S_{q}^{*}+I$ (see [20], also [3]).

We will actually work most of the time with the matrices of the above operators, with respect to the canonical basis. The reason for this is two-fold: on one hand, we will deal with infinite sums of monomials in $S_{q}$ and $S_{q}^{*}$ which don't make sense (are divergent) as operators, but still have meaning as matrices; on the other hand, when formulated in terms of matrices, all the considerations are also valid for $q=1$.

Hence, for every $q \in[0,1]$, we (also) denote by $S_{q}, S_{q}^{*}$, the matrices:

$$
\left(S_{q}\right)_{i, j}=\left\{\begin{array}{ll}
\sqrt{[i]_{q}}, & \text { if } i-j=1 \\
0, & \text { otherwise }
\end{array} ; \quad\left(S_{q}^{*}\right)_{i, j}=\left\{\begin{array}{ll}
\sqrt{[j]_{q}}, & \text { if } j-i=1 \\
0, & \text { otherwise }
\end{array} .\right.\right.
$$

These matrices belong to the algebra $\mathscr{M}$ of 1.1 . B. For $q \neq 1$, they are exactly the ones of the weighted shift $S_{q}$ and its adjoint, with respect to the canonical basis (it will always be clear from the context whether we are referring to the operator or to its matrix).

The main result of the paper can then be stated as follows.

1.2. Theorem. If $\theta(z)=\sum_{n=1}^{\infty} \alpha_{n} z^{n}$ is a formal power series vanishing at zero, and $q \in[0,1]$ is a parameter, then all the following constructions lead to the same distribution $\mu: \mathbf{C}\langle X\rangle \rightarrow \mathbf{C}$. 
(a) The moments of $\mu$ are given by the formula

$$
\mu\left(X^{n}\right)=\sum_{\substack{\pi \in \mathcal{P}(\{1, \ldots, n\}) \\ \pi=\left\{B_{1}, \ldots, B_{k}\right\}}} q^{c_{o}(\pi)} \prod_{j=1}^{k}\left[\left|B_{j}\right|-1\right]_{q} ! \alpha_{\left|B_{j}\right|}, \quad n \geqq 1 .
$$

$\left(c_{o}(\pi)\right.$ is as defined in (1.5), and the $\alpha$ 's are the coefficients of $\theta .\left|B_{j}\right|$ stands, of course, for the number of elements of $B_{j}$, and we use the customary notation for $q$-factorials: $[0]_{q} !=1,[l]_{q} !=[1]_{q}[2]_{q} \cdots[l]_{q}$ for $l \geqq 1$.)

(b) Consider the matrix

$$
T_{\theta, q}=S_{q}^{*}+\sum_{n=0}^{\infty} \alpha_{n+1} S_{q}^{n} \in \mathscr{M}
$$

(this makes sense, because any two of the matrices $S_{q}^{*}, I, S_{q}, S_{q}^{2}, \ldots$ involved in the sum are supported on different diagonals); define $\mu$ by

$$
\mu(f)=\omega\left(f\left(T_{\theta, q}\right)\right), \quad f \in \mathbf{C}\langle X\rangle,
$$

with $\omega$ the linear functional of (1.7). ${ }^{1}$

(c) Consider the lower triangular matrix $M(=M(\theta, q))$, with entries:

$$
(M)_{i, j}= \begin{cases}\alpha_{l-j}[i-1]_{q} ! /[j]_{q} !, & \text { if } i>j \\ 1, & \text { if } i=j \\ 0, & \text { if } i<j\end{cases}
$$

(in particular $M$ belongs to the algebra $\mathscr{M}$ of 1.1.B). There exists a unique matrix $\Gamma \in \mathscr{M}$ which is lower triangular with 1's on the diagonal, and which satisfies:

$$
\Gamma=(1 \oplus \Gamma) M .
$$

$\Gamma$ can be in fact written explicitly as a limit,

$$
\Gamma=\lim _{n \rightarrow \infty}(\underbrace{1 \oplus \cdots \oplus 1}_{n} \oplus M)(\underbrace{1 \oplus \cdots \oplus 1}_{n-1} \oplus M) \cdots(1 \oplus M) M,
$$

with the direct sums taken as in (1.8). Define $\mu$ to be the distribution with moments given by the entries of the first column of $\Gamma$ :

$$
\mu\left(X^{n}\right)=(\Gamma)_{n, 0}, \quad n \geqq 0 .
$$

1.3. Definition. Let $q \in[0,1]$ be a parameter. For every formal power series vanishing at zero, $\theta(z)=\sum_{n=1}^{\infty} \alpha_{n} z^{n}$, denote by $R_{q}^{-1}(\theta)$ the distribution $\mu$ constructed in Theorem 1.2. Consider the map $R_{q}^{-1}: \Theta \rightarrow \Sigma$ (with $\Theta, \Sigma$ as in the formulae (1.4), (1.1), respectively) which is obtained in this way. Then $R_{q}^{-1}$ is a bijection - as it is immediate from characterization (a) of 1.2, for instance; its inverse, $\left(R_{q}^{-1}\right)^{-1}: \Sigma \rightarrow \Theta$, will be denoted by $R_{q}$, and called $R_{q}$-transform.

1.4. Remark. (the case $q=0$ ). The description of $R_{q}^{-1}$ provided by (b) of Theorem 1.2 is a straightforward extension of the one used by Voiculescu in [17], for $q=0$;

\footnotetext{
1 The terminology used in such situations is that " $\mu$ is the distribution of $T_{\theta, q}$ in the noncommutative probability space $(\mathscr{M}, \omega) "$ - see [16], or the monograph [18].
} 
the only modification for arbitrary $q \in[0,1]$ is that there exists a weight on the shift. In particular, the $R_{0}$-transform of Definition 1.3 coincides with the $R$-transform of $[16,17]$.

Note also that, for $q=0$, Eq. (1.10) in (a) of Theorem 1.2 becomes:

$$
\mu\left(X^{n}\right)=\sum_{\substack{\pi \in \mathcal{N} \mathscr{C}(\{1, \ldots, n\}) \\ \pi=\left\{B_{1}, \ldots, B_{k}\right\}}} \alpha_{\left|B_{1}\right|} \ldots \alpha_{\left|B_{k}\right|}, \quad n \geqq 1
$$

where $\mathscr{N} \mathscr{C}(\{1, \ldots, n\})$ denotes the set of non-crossing partitions of $\{1, \ldots, n\}$. This relation between the moments of a distribution $\mu$ and the coefficients $\left(\alpha_{n}\right)_{n=1}^{\infty}$ of $R(\mu)$ was first remarked by R. Speicher [13].

1.5. Remark. (the case $q=1$ ). By putting $q=1$ in Eq. (1.10), and using the fact that for a partition $n=k_{1}+2 k_{2}+\cdots+n k_{n}\left(k_{1}, \ldots, k_{n} \geqq 0\right)$ of the number $n$ there are $n ! /\left[(1 !)^{k_{1}} \cdots(n !)^{k_{n}} k_{1} ! \cdots k_{n} !\right]$ partitions of $\{1, \ldots, n\}$ which have $k_{1}$ classes of 1 element, $\ldots, k_{n}$ classes of $n$ elements, we get:

$$
\frac{\mu\left(X^{n}\right)}{n !}=\sum_{\substack{k_{1}, \ldots, k_{n} \geqq 0 \\ k_{1}+2 k_{2}+\cdots+n k_{n}=n}} \frac{\left(\frac{\alpha_{1}}{1}\right)^{k_{1}} \cdots\left(\frac{\alpha_{n}}{n}\right)^{k n}}{k_{1} ! \cdots k_{n} !}, n \geqq 1
$$

in (1.17), $\mu$ is a distribution, and $\left(\alpha_{n}\right)_{n=1}^{\infty}$ are the coefficients of $R_{1}(\mu)$. But, as it is easily checked, (1.17) means that the series $\sum_{n=0}^{\infty} \frac{\mu\left(X^{n}\right)}{n !} z^{n}$ is the exponential of $\sum_{n=1}^{\infty} \frac{\alpha_{n}}{n} z^{n} ;$ this entails the formula

$$
\begin{aligned}
\left(R_{1}(\mu)\right)(z) & =-i z(\log \mathscr{F}(\mu))^{\prime}(-i z) \\
& =-i z(\mathscr{F}(\mu))^{\prime}(-i z) /(\mathscr{F}(\mu))(-i z)
\end{aligned}
$$

In particular, the $R_{1}$-transform of Definition 1.3 differs from the logarithm of the Fourier transform only by a linear bijective map from the space $\Theta$ of (1.4) onto itself; and, as a consequence, $R_{1}$ shares the property of $\log \mathscr{F}$ of linearizing the usual convolution of distributions.

1.6. Remark. (relation with continued fractions). We now look at the characterization (c) in Theorem 1.2. Let $q \in[0,1]$ be a parameter, let $\theta(z)=\sum_{n=1}^{\infty} \alpha_{n} z^{n}$ be a formal power series vanishing at zero, and define the lower triangular matrices $M, \Gamma$ as in (1.13), (1.14) of 1.2 (c). Equation (1.14) defining $\Gamma$ remains valid if we delete the first line of $\Gamma$, and also of $1 \oplus \Gamma$. Moreover, what is obtained from $1 \oplus \Gamma$ (by deleting the first line) has only zeros on the first column; hence we can delete that column too, if we also erase the first line of $M$. Making for $i>j$ the notation $(\Gamma)_{i, j}=\gamma_{i, j}($ the $(i, j)$-entry of $\Gamma)$, we thus arrive to:

$$
\left(\begin{array}{ccccc}
\gamma_{1,0} & 1 & & & \\
\gamma_{2,0} & \gamma_{2,1} & 1 & & \\
\gamma_{3,0} & \gamma_{3,1} & \gamma_{3,2} & 1 & \\
\gamma_{4,0} & \gamma_{4,1} & \gamma_{4,2} & \gamma_{4,3} & 1 \\
\cdots & \cdots & \ldots & \ldots & \ldots
\end{array}\right)=\left(\begin{array}{cccc}
1 & & & \\
\gamma_{1,0} & 1 & & \\
\gamma_{2,0} & \gamma_{2,1} & 1 & \\
\gamma_{3,0} & \gamma_{3,1} & \gamma_{3,2} & 1 \\
\ldots & \ldots & \ldots & \ldots
\end{array}\right)
$$




$$
\left(\begin{array}{ccccc}
\alpha_{1}[0]_{q} ! /[0]_{q} ! & 1 & & & \\
\alpha_{2}[1]_{q} ! /[0]_{q} ! & \alpha_{1}[1]_{q} ! /[1]_{q} ! & 1 & & \\
\alpha_{3}[2]_{q} ! /[0]_{q} ! & \alpha_{2}[2]_{q} ! /[1]_{q} ! & \alpha_{1}[2]_{q} ! /[2]_{q} ! & 1 & \\
\alpha_{4}[3]_{q} ! /[0]_{q} ! & \alpha_{3}[3]_{q} ! /[1]_{q} ! & \alpha_{2}[3]_{q} ! /[2]_{q} ! & \alpha_{1}[3]_{q} ! /[3]_{q} ! & 1 \\
\ldots & \ldots & \ldots & \ldots & \ldots
\end{array}\right)
$$

The latter equation is very similar (especially if we also take into account the significance of the first column of $\Gamma$ ) to what one has when converting a continued $J$-fraction into a power series, after the method of Stieltjes (see [19], Sect. 53). The difference between (1.19) and the matrix equation of Stieltjes is that the third matrix in (1.19) may have non-zero $(i, j)$-entries for all pairs $(i, j)$ such that $j \leqq i+1$ (and not only for those $(i, j)$ with $|i-j| \leqq 1)$.

Note that if the considered power series $\theta$ happens to be a quadratic polynomial vanishing at zero, $\theta(z)=\alpha_{1} z+\alpha_{2} z^{2}$ (i.e. $\alpha_{3}=\alpha_{4}=\cdots=0$ ), then the theorem of Stieltjes can indeed be applied, and gives

$$
\sum_{n=0}^{\infty} \mu\left(X^{n}\right) z^{n}=\frac{1}{1-\alpha_{1} z-\frac{[1]_{q} \alpha_{2} z^{2}}{1-\alpha_{1} z-\frac{[2]_{q} \alpha_{2} z^{2}}{1-\alpha_{1} z-\frac{[3]_{q} \alpha_{2} z^{2}}{1-\alpha_{1} z-\cdots}}}},
$$

with $\mu=R_{q}^{-1}(\theta)$. In particular, by putting $\alpha_{1}=0$ and $\alpha_{2}=1$ in (1.20), we have that the generating function for the moments of $R_{q}^{-1}\left(z^{2}\right)$ is the expansion of

$$
\frac{1}{1-\frac{[1]_{q} z^{2}}{1-\frac{[2]_{q} z^{2}}{1-\frac{[3]_{q} z^{2}}{1-\cdots}}}}
$$

This continued fraction is known to be associated to the $q$-continuous Hermite polynomials (see for instance [7], Eq. (4.4), or Sect. 2 and 3.5 of [1]); therefore, $R_{q}^{-1}\left(z^{2}\right)$ is the measure associated with this set of orthogonal polynomials.

We mention that various facts concerning the particular case discussed in the preceding paragraph were known. On one hand, the formula for the moment of order $2 n$ of $R_{q}^{-1}\left(z^{2}\right)$ provided by characterization (a) in 1.2 is $\Sigma_{\pi} q^{c_{o}(\pi)}$, with summation after all the matchings (i.e. partitions into classes with exactly two elements) of $\{1, \ldots, 2 n\}$. The relation between this sum and the continued fraction (1.21) was pointed out by Touchard [15] (see also Flajolet [5]). On the other hand, the distribution of the operator $S_{q}+S_{q}^{*}$ with respect to the functional $\omega$ (with $S_{q}, \omega$ as in Notations 1.1 above) was studied by Bożejko and Speicher [3] Part II, and its connection with the $q$-continuous Hermite polynomials was found. (In view of 1.2 (b) above, an alternative definition of $R_{q}^{-1}\left(z^{2}\right)$ is as the distribution of $S_{q}+S_{q}^{*}$ with respect to $\omega$.)

1.7. Remark. (equations in generating functions related to the $R_{q}$-transforms). We consider again Eq. (1.14) of Theorem 1.2(c), and we use the same notations $(q, \theta, M, \Gamma)$ as in the preceding remark. In addition, for every $j \geqq 0$ we denote

$$
\varphi_{j}(z)=\sum_{l=j}^{\infty} z^{l-\jmath}(\Gamma)_{i, j} ;
$$


since $\Gamma$ is lower triangular, $\varphi_{j}$ could be called "the generating function for the $j^{\text {th }}$ column of $\Gamma$."

Equation (1.14) is equivalent to the fact that (for every $j \geqq 0$ ) the $j^{\text {th }}$ column of $\Gamma$ is the linear combination of the columns of $1 \oplus \Gamma$, with coefficients taken from the $j^{\text {th }}$ column of $M$. Hence (1.14) comes to the system:

$$
\left\{\begin{aligned}
\varphi_{0}= & 1+\alpha_{1} z[0]_{q} ! \varphi_{0}+\alpha_{2} z^{2}[1]_{q} ! \varphi_{1}+\alpha_{3} z^{3}[2]_{q} ! \varphi_{2}+\cdots \\
z^{j} \varphi_{j}= & z^{j} \varphi_{j-1}+\alpha_{1} z^{j+1}\left([j]_{q} ! /[j]_{q} !\right) \varphi_{J}+\alpha_{2} z^{j+2}\left([j+1]_{q} ! /[j]_{q} !\right) \varphi_{J+1} \\
& +\alpha_{3} z^{j+3}\left([j+2]_{q} ! /[j]_{q} !\right) \varphi_{j+2}+\cdots, j \geqq 1 .
\end{aligned}\right.
$$

Renormalizing: $\psi_{j}=[j]_{q} ! \varphi_{j}, j \geqq 0$, we obtain:

$$
\left\{\begin{array}{l}
\psi_{0}=1+\alpha_{1} z \psi_{0}+\alpha_{2} z^{2} \psi_{1}+\alpha_{3} z^{3} \psi_{2}+\cdots \\
\psi_{j}=[j]_{q} \psi_{j-1}+\alpha_{1} z \psi_{j}+\alpha_{2} z^{2} \psi_{j+1}+\alpha_{3} z^{3} \psi_{j+2}+\cdots, j \geqq 1
\end{array}\right.
$$

Now, the first column of $\Gamma$ contains the moments of $\mu=R_{q}^{-1}(\theta)$ (by (c) of Theorem 1.2); hence $\psi_{0}=\phi_{0}$ is the generating function for the moments of $\mu, \psi_{0}=$ $\sum_{n=0}^{\infty} \mu\left(X^{n}\right) z^{n}$. If it were not for the other generating functions $\psi_{1}, \psi_{2}, \psi_{3}, \ldots$ which appear in (1.23), this system would thus express the relation between $\mu$ and $\theta$ (i.e. the $R_{q}$-transform) in terms of generating functions.

The method of handling $\psi_{1}, \psi_{2}, \psi_{3}, \ldots$ seems to consist in expressing them in terms of $\psi_{0}$. Their form can be guessed (and then proved) both for $q=0$ and $q=1$ (but we couldn't find it explicitly for arbitrary $q$ ). To be precise, we have:

$$
\left\{\begin{array}{cc}
\psi_{j}=\psi_{0}^{j+1}, j \geqq 1, & \text { for } q=0 \\
\psi_{j}=\left(z^{j} \psi_{0}\right)^{(j)}, j \geqq 1, & \text { for } q=1
\end{array}\right.
$$

(where $\left(z^{j} \psi_{0}\right)^{(j)}$ stands for the $j^{\text {th }}$ derivative of $z^{j} \psi_{0}$ ). Proving (1.24) is very easy - let us show it for instance for $q=0$. From the fact that the $R_{q}$-transform is welldefined it follows that the system (1.23) has a unique solution, where $\psi_{0}$ is given as data, and $\left(\alpha_{j}\right)_{j=1}^{\infty}$ and $\left(\psi_{j}\right)_{j=1}^{\infty}$ are viewed as unknowns. So, if we can construct (for $q=0$ ) a solution of (1.23) which has $\psi_{j}=\psi_{0}^{j+1}, j \geqq 1$, then the first relation (1.24) will be proved. But we can proceed as follows: since the constant term of $\psi_{0}$ is 1 , it is clear that there are unique $\alpha_{1}, \alpha_{2}, \alpha_{3}, \ldots \in \mathbf{C}$ such that

$$
\psi_{0}=1+\alpha_{1} z \psi_{0}+\alpha_{2} z^{2} \psi_{0}^{2}+\alpha_{3} z^{3} \psi_{0}^{3}+\cdots
$$

These $\left(\alpha_{j}\right)_{j=1}^{\infty}$, together with $\left(\psi_{j}=\psi_{0}^{j+1}\right)_{j=0}^{\infty}$ satisfy hence the first equation in (1.23); but multiplying (1.25) with $\psi_{0}, \psi_{0}^{2}, \psi_{0}^{3}, \ldots$ it is actually clear that not only the first, but all the equations of (1.23) (for $q=0$ ) are satisfied, and we are done.

We note that (1.25) is equivalent to Theorem 2.9 of [17]. The analogue of all this for $q=1$ leads to nothing else but the second form of the equation (1.18).

The paper is divided into sections as follows: after a few combinatorial preliminaries described in Sect. 2, the proof of the main result of the paper, Theorem 1.2, is presented in Sect. 3. In the fourth (and last) section we consider the convolution laws $\bigoplus_{q}, 0 \leqq q \leqq 1$, which are linearized by the $R_{q}$-transforms, and (without going too deep in their study) we point out some basic properties which are built in their definition. 


\section{Combinatorial Preliminaries}

2.1 Notations Let $n$ be a positive integer. We denote by $\mathscr{C}_{n}$ the set of $n$-tuples ${ }^{2}$ $\left\{\varepsilon=\left(\varepsilon_{1}, \ldots, \varepsilon_{n}\right) \mid \varepsilon_{1}, \ldots, \varepsilon_{n} \in \mathbf{N} \cup\{-1\}, \sum_{j=1}^{m} \varepsilon_{j} \geqq 0\right.$ for every $\left.1 \leqq m \leqq n, \sum_{j=1}^{n} \varepsilon_{j}=0\right\}$.

We denote by $\rho_{n}: \mathscr{P}(\{1, \ldots, n\}) \rightarrow \mathscr{C}_{n}$ the map which associates to the partition $\pi=\left\{B_{1}, \ldots, B_{k}\right\} \in \mathscr{P}(\{1, \ldots, n\})$ the $n$-tuple $\varepsilon=\left(\varepsilon_{1}, \ldots, \varepsilon_{n}\right) \in \mathscr{C}_{n}$ given by:

$$
\varepsilon_{m}= \begin{cases}\left|B_{j}\right|-1, & \text { if } m=\min B_{j} \text { for some (uniquely determined) } 1 \leqq j \leqq k, \\ -1, & \text { otherwise }\end{cases}
$$

An important ingredient in the proof of $(a) \Leftrightarrow(b)$ in Theorem 1.2 is the combinatorial identity stated as follows:

2.2 Theorem. Let $n$ be a positive integer, and let $q \in[0,1]$ be a parameter. Then for every $\varepsilon=\left(\varepsilon_{1}, \ldots, \varepsilon_{n}\right) \in \mathscr{C}_{n}$ we have:

$$
\sum_{\pi \in \rho_{n}^{-1}(\varepsilon)} q^{c_{0}(\pi)}=\left(\prod_{\substack{1 \leq m \leq n \\ \text { such that } \\ \varepsilon_{m}=-1}}\left[\varepsilon_{1}+\cdots+\varepsilon_{m-1}\right]_{q}\right) \cdot\left(\prod_{\substack{1 \leq m \leq n \\ \text { such that } \\ \varepsilon_{m} \geqq 0}}\left[\varepsilon_{m}\right]_{q} !\right)^{-1}
$$

$\left(\rho_{n}: \mathscr{P}(\{1, \ldots, n\}) \rightarrow \mathscr{C}_{n}\right.$ as in $2.1, c_{o}(\cdot)$ as in 1.1.A).

$n, q$, and $\varepsilon=\left(\varepsilon_{1}, \ldots, \varepsilon_{n}\right) \in \mathscr{C}_{n}$ about which we are proving (2.3) are fixed for the rest of the section. Let the set of positions $\left\{m \mid 1 \leqq m \leqq n, \varepsilon_{m} \geqq 0\right\}$ be written explicitly as $\left\{p_{1}, \ldots, p_{k}\right\}$, with $1=p_{1}<\cdots<p_{k} \leqq n$. We also use the shorthand notation $\varepsilon_{p_{J}}=l_{j}, 1 \leqq j \leqq k$; note that

$$
l_{1}+\cdots l_{k}=n-k
$$

(indeed, we have $0=\varepsilon_{1}+\cdots+\varepsilon_{n}=\varepsilon_{p_{1}}+\cdots \varepsilon_{p_{k}}+\underbrace{(-1)+\cdots(-1)}_{n-k}=l_{1}+\cdots+$ $\left.l_{k}-(n-k)\right)$.

For every $1 \leqq j \leqq k$ we denote by $s_{j}$ the sum:

$$
s_{J}=\sum_{m=1}^{p_{J}} \varepsilon_{m}=\left[\sum_{l=1}^{j}\left(l_{i}+1\right)\right]-p_{j}=\left(n-p_{J}\right)-\left[\sum_{i=J+1}^{k}\left(l_{l}+1\right)\right] ;
$$

$s_{j}$ is a non-negative integer, and in fact $s_{j} \geqq l_{j}, 1 \leqq j \leqq k\left(s_{J}=\left(\sum_{m=1}^{p_{J}-1} \varepsilon_{m}\right)+\right.$ $\left.\varepsilon_{p_{J}} \geqq 0+\varepsilon_{p_{J}}=l_{J}\right)$.

2.3 Lemma. There exists a bijection between the index $\operatorname{set} \rho_{n}^{-1}(\varepsilon)$ of the sum (2.3), and the set

$$
\left\{\left(A_{1}, \ldots, A_{k}\right)\left|A_{J} \subseteq\left\{1, \ldots, s_{j}\right\},\right| A_{j} \mid=l_{j} \text { for every } 1 \leqq j \leqq k\right\},
$$

\footnotetext{
2 One thinks of the elements of $\mathscr{C}_{n}$ as of paths in the square lattice, by identifying $\left(\varepsilon_{1}, \ldots, \varepsilon_{n}\right)$ with the sequence of points $(0,0),\left(1, \varepsilon_{1}\right),\left(2, \varepsilon_{1}+\varepsilon_{2}\right), \ldots,\left(n, \varepsilon_{1}+\cdots+\varepsilon_{n}\right)=(n, 0)$.
} 
such that the following holds: if $\pi \in \rho_{n}^{-1}(\varepsilon)$ corresponds to $\left(A_{1}, \ldots, A_{k}\right)$ in the set (2.6), then

$$
c_{o}(\pi)=c_{o}\left(A_{1}\right)+\cdots c_{o}\left(A_{k}\right) \text {, }
$$

where for any finite set of positive integers $A=\left\{a_{1}, \ldots, a_{h}\right\}$ we put

$$
\begin{aligned}
c_{o}(A) & =\left(a_{1}-1\right)+\left(a_{2}-2\right)+\cdots+\left(a_{h}-h\right) \\
& =\text { card }\{(b, a) \mid a, b \text { positive integers, } b<a, a \in A, b \notin A\} .
\end{aligned}
$$

Proof. Given a $k$-tuple of sets $\left(A_{1}, \ldots, A_{k}\right)$ as in (2.6), we construct a partition $\pi=\left\{B_{1}, \ldots, B_{k}\right\} \in \rho_{n}^{-1}(\varepsilon)$ as follows.

We begin by taking $p_{1} \in B_{1}, \ldots, p_{k} \in B_{k} ; p_{J}$ must come out to be in fact the minimal element of $B_{j}, 1 \leqq j \leqq k$, and we also know that $B_{j} \backslash\left\{p_{j}\right\}$ must contain exactly $l_{j}$ elements.

The next step in constructing $\pi$ is to choose the $l_{k}$ elements which form $B_{k} \backslash\left\{p_{k}\right\}$. All these $l_{k}$ elements must lie to the right of $p_{k}$. There are exactly $n-p_{k}=s_{k}$ numbers to the right of $p_{k}$ in $\{1, \ldots, n\}$, and our way of choosing $l_{k}$ of them (to form $B_{k} \backslash\left\{p_{k}\right\}$ ) is the one dictated by $A_{k}$. (Recall that $A_{k}$, the last set in the $k$-tuple $\left(A_{1}, \ldots, A_{k}\right)$ we started with, is a subset with $l_{k}$ elements of $\left\{1, \ldots, s_{k}\right\}$; therefore, whenever we have a totally ordered set of $s_{k}$ elements, $A_{k}$ indicates a choice of $l_{k}$ of them, in the obvious way.)

We now go to the choice of the $l_{k-1}$ elements which form $B_{k-1} \backslash\left\{p_{k-1}\right\}$. All these $l_{k-1}$ elements must lie to the right of $p_{k-1}$, and we must take care that none of them falls in the (already chosen) block $B_{k}$. There are $n-p_{k-1}$ numbers to the right of $p_{k-1}$ in $\{1, \ldots, n\}$, and $l_{k}+1$ of them are already picked in $B_{k}$, therefore we have to choose our $l_{k-1}$ numbers out of $\left(n-p_{k-1}\right)-\left(l_{k}+1\right)=s_{k-1}$ possibilities. We make the choice as dictated by the set $A_{k-1}$ in the $k$-tuple $\left(A_{1}, \ldots, A_{k}\right)$ we started with.

We continue by repeating the algorithm described in the preceding paragraph, in order to choose, recursively, the $l_{k-2}$ elements of $B_{k-2} \backslash\left\{p_{k-2}\right\}, \ldots$, the $l_{1}$ elements of $B_{1} \backslash\left\{p_{1}\right\}$. This leads us to the partition $\pi=\left\{B_{1}, \ldots, B_{k}\right\} \in \rho_{n}^{-1}(\varepsilon)$ we needed to construct.

Let us remark that the partition $\pi \in \rho_{n}^{-1}(\varepsilon)$ we arrived to satisfies (2.7). Indeed, the formula (1.5) defining $c_{o}(\pi)$ can also be written:

$$
c_{o}(\pi)=\sum_{j=1}^{k} \operatorname{card}\left\{\begin{array}{ll}
\left(m_{1}, m_{2}, m_{3}, m_{4}\right) & \begin{array}{l}
\mid 1 \leqq m_{1}<m_{2}<m_{3}<m_{4} \leqq n \\
\mid m_{1} \sim m_{3}, m_{2} \sim m_{4}, \\
\mid m_{2}=p_{j}, m_{1}=p_{i} \text { for some } i<j
\end{array}
\end{array}\right\}
$$

(this is because, obviously, for every 4-tuple $\left(m_{1}, m_{2}, m_{3}, m_{4}\right)$ appearing in (1.5), $m_{1}$ and $m_{2}$ must lie in $\left.\left\{p_{1}, \ldots, p_{k}\right\}\right)$. Now, a 4-tuple appearing in (2.9) is clearly determined by its third and fourth components, which shows that for a given $1 \leqq$ $j \leqq k$, the set appearing on the right-hand side of (2.9) is naturally in bijection with

$$
\left\{(b, a) \mid p_{j}<b<a, a \in B_{j}, b \in \bigcup_{i=1}^{j-1} B_{l}\right\} ;
$$

but by comparing (2.10) with (2.8), one sees without difficulty that the cardinality of the set $(2.10)$ equals $c_{o}\left(A_{j}\right)$. Hence the $j^{\text {th }}$ term of the sum (2.9) is $c_{o}\left(A_{j}\right)$, which establishes (2.7) 
Finally, it is an easy exercise, left to the reader, to check that the construction described in the first five paragraphs of the proof does indeed establish a bijection between the set of $k$-tuples $(2.6)$ and $\rho_{n}^{-1}(\varepsilon)$. QED

We also record the following known fact (see for instance [6], Lemma 5.1).

2.4. Lemma. For $l \leqq s$ non-negative integers we have

$$
\sum_{\substack{A \subseteq\{1, \ldots s\} \\
s . t|A| A \mid=i}} q^{c_{o}(A)}=\left[\begin{array}{l}
s \\
l
\end{array}\right]_{q}\left(\stackrel{\text { def }}{=} \frac{[s]_{q} !}{[l]_{q} ![s-l] q !}\right) .
$$

2.5. Proof of Theorem 2.2. We have:

$$
\begin{aligned}
\sum_{\pi \in \rho_{n}^{-1}(\varepsilon)} q^{c_{o}(\pi)} & =\sum_{\substack{\left(A_{1}, \ldots, A_{k}\right) \\
\text { as in }(2,6)}} q^{c_{O}\left(A_{1}\right)+\cdots+c_{o}\left(A_{k}\right)} \quad \text { (by Lemma 2.3) } \\
& =\prod_{j=1}^{k}\left(\sum_{\substack{A_{j} \subseteq\left\{1, \ldots, s_{J}\right\} \\
\mathrm{st}\left|A_{j}\right|=l_{j}}} q^{c_{o}\left(A_{j}\right)}\right)=\prod_{j=1}^{k}\left[\begin{array}{c}
s_{j} \\
l_{j}
\end{array}\right]_{q} \quad(\text { by Lemma 2.4) } \\
& =\frac{\left[s_{1}\right]_{q} ! \cdots\left[s_{k}\right]_{q} !}{\left[s_{1}-l_{1}\right]_{q} ! \cdots\left[s_{k}-l_{k}\right]_{q} !} \cdot \frac{1}{\left[l_{1}\right]_{q} ! \cdots\left[l_{k}\right]_{q} !}
\end{aligned}
$$

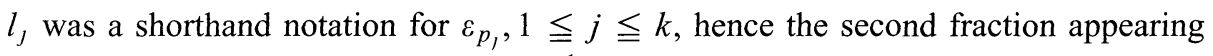
in (2.12) is exactly $\left(\prod_{\substack{1 \leq m \leq n \\ \text { s.t } \varepsilon_{m} \geqq 0}}\left[\varepsilon_{m}\right]_{q} !\right)^{-1}$. In order to establish (2.3) it remains to verify that

$$
\frac{\left[s_{1}\right]_{q} ! \cdots\left[s_{k}\right]_{q} !}{\left[s_{1}-l_{1}\right]_{q} ! \cdots\left[s_{k}-l_{k}\right]_{q} !}=\prod_{\substack{1 \leq m \leq n \\ \text { such =hat } \\ \varepsilon m=-1}}\left[\varepsilon_{1}+\cdots+\varepsilon_{m-1}\right]_{q} .
$$

But it is easily seen that

$$
\left\{\begin{array}{l}
\prod_{m=p_{1}+1}^{p_{J+1}-1}\left[\varepsilon_{1}+\cdots+\varepsilon_{m-1}\right]_{q}=\frac{\left[s_{j}\right] q_{q} !}{\left[s_{J+1}-l_{J+1}\right]_{q} !}, \quad 1 \leqq j \leqq k-1 . \\
\prod_{m=p_{k}+1}^{n}\left[\varepsilon_{1}+\cdots+\varepsilon_{m-1}\right]_{q}=\left[s_{k}\right]_{q} !
\end{array}\right.
$$

Multiplying the equalities (2.14) together, and taking into account that $s_{1}=l_{1}$ yields (2.13) and concludes the proof. QED

2.6. Corollary. For $n, q$ and $\varepsilon=\left(\varepsilon_{1}, \ldots, \varepsilon_{n}\right) \in \mathscr{C}_{n}$ as in Theorem 2.2 we have:

$$
\sum_{\substack{\pi \in \rho_{n}^{-1}(\varepsilon) \\ \pi=\left\{B_{1}, \ldots, B_{k}\right\}}} q^{c_{o}(\pi)} \prod_{j=1}^{k}\left[\left|B_{j}\right|-1\right]_{q} !=\prod_{\substack{1 \leq m \leq n \\ \text { such that } \\ \varepsilon_{m}=-1}}\left[\varepsilon_{1}+\cdots+\varepsilon_{m-1}\right]_{q} .
$$

Proof. From the definition of the map $\rho_{n}: \mathscr{P}(\{1, \ldots, n\}) \rightarrow \mathscr{C}_{n}$ it is immediate that, for every $\pi=\left\{B_{1}, \ldots, B_{k}\right\} \in \rho_{n}^{-1}(\varepsilon)$, we have $\prod_{j=1}^{k}\left[\left|B_{j}\right|-1\right]_{q} !=\prod_{\substack{1 \leq m \leq n \\ \text { s.t. } \varepsilon_{m} \geqq 0}}\left[\varepsilon_{m}\right]_{q}$ !; hence (2.15) reduces to (2.3). QED 
2.7. Remark. The relatively short derivation of (2.3) presented above was suggested to us by an anonymous referee for a talk related to this paper (at a conference on algebraic combinatorics, Rutgers, May 1994). We mention that an alternative proof of the identity (2.15) (hence, equivalently, of (2.3)) can be made by using an argument which enumerates a certain class of inversions of permutations of $\{1, \ldots, n\}, n \geqq 1$. This alternative proof is presented in [10], Sect. 4; it is sensibly longer, but offers on the other hand a more complete picture of the situation. The $q$ factorials in (2.15) come out, during the derivation via permutations, as sums of the form $\Sigma_{\tau \in \mathscr{S}_{m}} q^{i n v(\tau)}$, where $\mathscr{S}_{m}$ denotes the group of all permutations of $\{1, \ldots, m\}$, and $\operatorname{inv}(\tau)$ is the number of inversions of $\tau \in \mathscr{S}_{m}$. (As it is well-known, the latter sum equals $[m]_{q}$ !-see for instance [14], Corollary 1.3.10.)

\section{The Proof of the Theorem 1.2}

In this section we prove the equivalence of (a), (b) and (c) in the main result of the paper (Theorem 1.2). We will fix throughout the whole section a formal power series vanishing at zero, $\theta(z)=\sum_{n=1}^{\infty} \alpha_{n} z^{n}$, and a value of the parameter $q \in[0,1]$. We start with the construction made in (b) of Theorem 1.2; that is, we consider the matrix $T_{\theta, q}$ defined by the formula (1.11), $T_{\theta, q}=S_{q}^{*}+\sum_{n=0}^{\infty} \alpha_{n+1} S_{q}^{n}$, and the distribution $\mu: \mathbf{C}\langle X\rangle \rightarrow \mathbf{C}$ defined by the formula (1.12), $\mu(f) \equiv \omega\left(f\left(T_{\theta, q}\right)\right)$ for $f \in \mathbf{C}\langle X\rangle$. We will have to show that the result of the constructions made in (a) and (c) of Theorem 1.2 is also $\mu$.

Let us make first a preliminary remark about the weighted shift $S_{q}$.

3.1. Remark. The calculations related to $S_{q}$ are usually simplified when we conjugate with certain diagonal matrices. More precisely, for $q \in[0,1]$, let us denote by $\Delta_{q}$ the diagonal matrix having $\sqrt{[i]_{q} !}$ on its $(i, i)$-entry, for every $i \geqq 0$. Then, with $S_{q}$ and $S_{q}^{*}$ as in the formulae (1.9) of 1.1.C, it is easily checked that:

$$
\left(\Delta_{q}^{-1} S_{q} \Delta_{q}\right)_{i, j}= \begin{cases}1, & \text { if } i=j+1 \\ 0, & \text { otherwise }\end{cases}
$$

(i.e. $\Delta_{q}^{-1} S_{q} \Delta_{q}$ is $S_{0}$, the usual unilateral shift); and

$$
\left(\Delta_{q}^{-1} S_{q}^{*} \Delta_{q}\right)_{i, j}= \begin{cases}{[j]_{q},} & \text { if } j=i+1 \\ 0, & \text { otherwise }\end{cases}
$$

Another fact which should be kept in mind is that conjugating with $\Delta_{q}$ preserves the linear functional $\omega: \mathscr{M} \rightarrow \mathbf{C}$ of $(1.7)$ - i.e. $\omega\left(\Delta_{q}^{-1} N \Delta_{q}\right)=\omega(N)$ for every $N \in$ $\mathscr{M}$ (immediate verification).

We start towards the proof of $(a) \Leftrightarrow(b)$ with the following

3.2. Lemma. Let $S_{q}, S_{q}^{*}$ be the matrices discussed in Remark 3.1, let $n$ be a positive integer, and let $\varepsilon_{1}, \ldots, \varepsilon_{n}$ be in $\mathbf{N} \cup\{-1\}$. Consider the monomial $S_{q}^{\varepsilon_{n}} S_{q}^{\varepsilon_{n-1}} \cdots S_{q}^{\varepsilon_{1}}$, where we make the convention that $S_{q}^{\varepsilon_{m}}$ means $S_{q}^{*}$ whenever $\varepsilon_{m}=-1$. (In the case when $\varepsilon_{m} \geqq 0, S_{q}^{\varepsilon_{m}}$ is of course the corresponding power of $S_{q}$ in the algebra $\mathscr{M}$ 
of 1.1.B; the monomial under consideration will hence also be an element of the same algebra.) We have:

$$
\omega\left(S_{q}^{\varepsilon_{n}} S_{q}^{\varepsilon_{n-1}} \cdots S_{q}^{\varepsilon_{1}}\right)= \begin{cases}\prod_{\substack{1 \leq m \leqq n \\ \text { st...m=-1}}}\left[\varepsilon_{1}+\cdots+\varepsilon_{m-1}\right]_{q}, & \text { if }\left(\varepsilon_{1}, \ldots, \varepsilon_{n}\right) \in \mathscr{C}_{n} \\ 0, & \text { otherwise }\end{cases}
$$

where $\omega: \mathscr{M} \rightarrow \mathbf{C}$ is the linear functional in (1.7) of 1.1.B, and $\mathscr{C}_{n}$ is the set defined in 2.1 .

Proof. The left-hand side of (3.3) equals, by Remark 3.1: $\omega\left(\left(\Delta_{q}^{-1} S_{q}^{\varepsilon_{n}} \Delta_{q}\right)\right.$ $\left(\Delta_{q}^{-1} S_{q}^{\varepsilon_{n-1}} \Delta_{q}\right) \cdots\left(\Delta_{q}^{-1} S_{q}^{\varepsilon_{1}} \Delta_{q}\right)$ ). This is (if we take into account that $\omega$ simply "takes the $(0,0)$-entry"):

$$
\sum_{i_{1}, \ldots, i_{n-1}=0}^{\infty}\left(\Delta_{q}^{-1} S_{q}^{\varepsilon_{n}} \Delta_{q}\right)_{0, l_{n-1}}\left(\Delta_{q}^{-1} S_{q}^{\varepsilon_{n-1}} \Delta_{q}\right)_{l_{n-1}, l_{n-2}} \cdots\left(\Delta_{q}^{-1} S_{q}^{\varepsilon_{2}} \Delta_{q}\right)_{l_{2}, l_{1}}\left(\Delta_{q}^{-1} S_{q}^{\varepsilon_{1}} \Delta_{q}\right)_{l_{1}, 0}
$$

Now from Remark 3.1 it is immediate that the entry-wise description of $\Delta_{q}^{-1} S_{q}^{\varepsilon} \Delta_{q}, \varepsilon \in$ $\mathbf{N} \cup\{-1\}$, is

$$
\left(S_{q}^{\varepsilon}\right)_{l, j}= \begin{cases}1, & \text { if } i-j=\varepsilon \in \mathbf{N} \\ {[j]_{q},} & \text { if } i-j=\varepsilon=-1 \\ 0, & \text { otherwise. }\end{cases}
$$

In order that an $(n-1)$-tuple $\left(i_{1}, \ldots, i_{n-1}\right)$ gives a non-zero contribution in the sum (3.4), we need therefore to have $0-i_{n-1}=\varepsilon_{n}, i_{n-1}-i_{n-2}=\varepsilon_{n-1}, \ldots, i_{2}-i_{1}=$ $\varepsilon_{2}, i_{1}-0=\varepsilon_{1}$; these relations come to $i_{m}=\sum_{k=1}^{m} \varepsilon_{k}, 1 \leqq m \leqq n-1$, together with $\sum_{k=1}^{n} \varepsilon_{k}=0$. Since $i_{1}, \ldots, i_{n-1}$ must be positive, it follows that we need in particular to have $\left(\varepsilon_{1}, \ldots, \varepsilon_{n}\right) \in \mathscr{C}_{n}$. Conversely, if $\left(\varepsilon_{1}, \ldots, \varepsilon_{n}\right) \in \mathscr{C}_{n}$, then the sum (3.4) will reduce to its term:

$$
\begin{gathered}
\left(\Delta_{q}^{-1} S_{q}^{\varepsilon_{n}} \Delta_{q}\right)_{0, \varepsilon_{1}+\cdots \varepsilon_{n-1}}\left(\Delta_{q}^{-1} S_{q}^{\varepsilon_{n-1}} \Delta_{q}\right)_{\varepsilon_{1}+\cdots \varepsilon_{n-1}, \varepsilon_{1}+\cdots \varepsilon_{n-2}} \cdots \\
\cdots\left(\Delta_{q}^{-1} S_{q}^{\varepsilon_{2}} \Delta_{q}\right)_{\varepsilon_{1}+\varepsilon_{2}, \varepsilon_{1}}\left(\Delta_{q}^{-1} S_{q}^{\varepsilon_{1}} \Delta_{q}\right)_{\varepsilon_{1}, 0}
\end{gathered}
$$

which (by (3.5)) is exactly the product in the right-hand side of (3.3). QED

3.3. The proof of $(a) \Leftrightarrow(b)$ in Theorem 1.2. Let us also fix a positive integer $n$; we have to show that an explicit formula for the $n^{\text {th }}$ moment of the distribution $\mu$ (considered in the first paragraph of this section) is provided by the right-hand side of Eq. (1.10). Recall that the definition we are currently having for the $n^{\text {th }}$ moment of $\mu$ reads: " $\mu\left(X^{n}\right)=\omega\left(T_{\theta, q}^{n}\right)$ ".

We make the notation $T_{\theta, q, n}=S_{q}^{*}+\sum_{m=0}^{n-1} \alpha_{m+1} S_{q}^{m}$. This is a truncation of $T_{\theta, q}$, in the sense that

$$
\left(T_{\theta, q, n}\right)_{i, j}= \begin{cases}\left(T_{\theta, q}\right)_{l, j}, & \text { if } i-j \leqq n-1 \\ 0, & \text { otherwise }\end{cases}
$$

(immediate verification). We claim that $\omega\left(T_{\theta, q}^{n}\right)=\omega\left(T_{\theta, q, n}^{n}\right)$. Indeed, we have:

$$
\omega\left(T_{\theta, q}^{n}\right)=\left(T_{\theta, q}^{n}\right)_{0,0}=\sum_{i_{1}, \ldots, i_{n-1}=0}^{\infty}\left(T_{\theta, q}\right)_{0, i_{n-1}}\left(T_{\theta, q}\right)_{l_{n-1}, i_{n-2}} \cdots\left(T_{\theta, q}\right)_{i_{2}, l_{1}}\left(T_{\theta, q}\right)_{l_{1}, 0}
$$


The diagonal height (defined as in 1.1.B) of $T_{\theta, q}$ is, obviously, equal to 1 ; hence every $(n-1)$-tuple $\left(i_{1}, \ldots, i_{n-1}\right)$ which gives a non-zero term of the sum in (3.7) must satisfy: $i_{n-1} \leqq 0+1, i_{n-2} \leqq i_{n-1}+1, \ldots, i_{1} \leqq i_{2}+1$. By taking (3.6) into account, it is then immediate that the entries of $T_{\theta, q}$ appearing in (3.7) can be replaced by the corresponding entries of $T_{0, q, n}$; in this way, the right-hand side of (3.7) is transformed into $\left(T_{\theta, q, n}^{n}\right)_{0,0}=\omega\left(T_{\theta, q, n}^{n}\right)$.

So we also have $\mu\left(X^{n}\right)=\omega\left(T_{\theta, q, n}^{n}\right)$, and this gives, by expanding $T_{\theta, q, n}^{n}$ as a sum:

$$
\sum_{\varepsilon_{1}, \ldots, \varepsilon_{n} \in\{-1,0,1, \ldots, n-1\}}\left(\omega\left(S_{q}^{\varepsilon_{n}} S_{q}^{\varepsilon_{n-1}} \cdots S_{q}^{\varepsilon_{1}}\right) \cdot \prod_{\substack{1 \leqq m \leqq n \\ \text { s.t. } \varepsilon_{m} \geqq 0}} \alpha_{1+\varepsilon_{m}}\right) .
$$

By also making use of Lemma 3.2, we thus arrive to

$$
\mu\left(X^{n}\right)=\sum_{\underline{\varepsilon}=\left(\varepsilon_{1}, \ldots, \varepsilon_{n}\right) \in \mathscr{C}_{n}}\left(\prod_{\substack{1 \leqq m \leqq n \\ s t}}\left[\varepsilon_{1}+\cdots \varepsilon_{m-1}\right]_{q} \cdot \prod_{\substack{1 \leqq m \leqq n \\ \text { s.t. } \varepsilon_{m} \geqq 0}} \alpha_{1+\varepsilon_{m}}\right) .
$$

But now, from Corollary 2.6 we know that the first product appearing on the right-hand side of (3.8) can be replaced with

$$
\sum_{\substack{\pi \in \rho_{n}^{-1}(\underline{\varepsilon}) \\ \pi=\left\{B_{1}, \ldots, B_{k}\right\}}} q^{c_{o}(\pi)} \prod_{j=1}^{k}\left[\left|B_{j}\right|-1\right]_{q} ! .
$$

By reviewing the definition of the map $\rho_{n}: \mathscr{P}(\{1, \ldots, n\}) \rightarrow \mathscr{C}_{n}$ (Eq. (2.2) in 2.1), one sees immediately that the second product on the right-hand side of (3.8) can be replaced with $\prod_{j=1}^{k} \alpha_{\left|B_{j}\right|}$, for an arbitrary $\pi \in \rho_{n}^{-1}(\underline{\varepsilon})$. When these replacements are performed, (3.8) becomes:

$$
\mu\left(X^{n}\right)=\sum_{\underline{\varepsilon} \in \mathscr{C}_{n}}\left(\sum_{\substack{\pi \in \rho_{n}^{-1}(\underline{\varepsilon}) \\ \pi=\left\{B_{1}, \ldots, B_{k}\right\}}} q^{c_{o}(\pi)} \prod_{j=1}^{k}\left[\left|B_{j}\right|-1\right]_{q} ! \prod_{j=1}^{k} \alpha_{\left|B_{j}\right|}\right),
$$

which is exactly (1.10). QED

Before going to the proof of $(b) \Leftrightarrow(c)$ in Theorem 1.2 , let us clarify the relation between Eqs. (1.14) and (1.15) appearing in 1.2 (c).

3.4. Lemma. Let $N$ be a lower triangular infinite matrix, with 1's on the principal diagonal. There exists a unique invertible matrix $X$ in the algebra $\mathscr{M}$ of 1.1.B, which satisfies

$$
X=(1 \oplus X) N
$$

(with $1 \oplus X$ defined as in (1.8) above). $X$ is also lower triangular with 1 's on the diagonal, and can in fact be written "explicitly" in the form:

$$
X=\lim _{n \rightarrow \infty}(\underbrace{1 \oplus \cdots \oplus 1}_{n} \oplus N)(\underbrace{1 \oplus \cdots \oplus 1}_{n-1} \oplus N) \cdots(1 \oplus N) N .
$$

Another explicit description of $X$ is the following: let $\breve{N} \in \mathscr{M}$ be the matrix obtained from $N$ by deleting the first line $\left((\check{N})_{i, j}=(N)_{i+1, j}, i, j \geqq 0\right)$. Then the entries of $X$ are:

$$
(X)_{l, j}=\left((\check{N})^{i}\right)_{0, j}, i, j \geqq 0 .
$$


Proof. The limit in (3.10) exists, because multiplying an arbitrary matrix $P \in \mathscr{M}$ with $\underbrace{1 \oplus \cdots \oplus 1}_{n} \oplus N$ on the left leaves unchanged the first $n+1$ lines of $P$ (hence,

for given $i, j \geqq 0$, all the matrix-products appearing in (3.10) are having from a certain moment the same $(i, j)$-entry).

If $X$ is the limit in (3.10), it is clear that $X$ is lower triangular with 1 's on the diagonal; in particular, $X$ is invertible. By adding 1 in direct sum, on the left of (3.10), we obtain:

$$
1 \oplus X=\lim _{n \rightarrow \infty}(\underbrace{1 \oplus \cdots \oplus 1}_{n} \oplus N)(\underbrace{1 \oplus \cdots \oplus 1}_{n-1} \oplus N) \cdots(1 \oplus N)
$$

which immediately implies that $X$ satisfies (3.9).

Conversely, let $Y \in \mathscr{M}$ be an invertible matrix such that $Y=(1 \oplus Y) N$. By adding 1's in direct sum, on the left of this equality, we get:

$$
\left\{\begin{array}{l}
Y=(1 \oplus Y) N \\
1 \oplus Y=(1 \oplus 1 \oplus Y)(1 \oplus N) \\
\cdots \cdots \cdots \cdots \cdots \\
\underbrace{1 \oplus \cdots \oplus 1}_{n} \oplus Y=(\underbrace{1 \oplus \cdots \oplus 1}_{n+1} \oplus Y)(\underbrace{1 \oplus \cdots \oplus 1}_{n} \oplus N)
\end{array}\right.
$$

that is

$$
\left\{\begin{array}{l}
(1 \oplus Y)^{-1} Y=N \\
(1 \oplus 1 \oplus Y)^{-1}(1 \oplus Y)=(1 \oplus N) \\
\cdots \cdots \cdots \cdots \cdots \\
(\underbrace{1 \oplus \cdots \oplus 1}_{n+1} \oplus Y)^{-1}(\underbrace{1 \oplus \cdots \oplus 1}_{n} \oplus Y)=(\underbrace{1 \oplus \cdots \oplus 1}_{n} \oplus N) \\
\ldots \ldots \cdots \cdots
\end{array}\right.
$$

Multiplying together the first $n+1$ equalities (3.12), in reversed order, we obtain:

$$
Y=(\underbrace{1 \oplus \cdots \oplus 1}_{n+1} \oplus Y)(\underbrace{1 \oplus \cdots \oplus 1}_{n} \oplus N) \cdots(1 \oplus N) N .
$$

Then letting $n \rightarrow \infty$ in (3.13) (and taking into account that $(\underbrace{1 \oplus \cdots \oplus 1}_{n+1} \oplus Y)$ tends for $n \rightarrow \infty$ to the identity matrix), it becomes clear that $Y$ coincides with $X$ of (3.10).

It remains to prove (3.11). While this can be done without difficulty from (3.10), let us remark that another simple way of doing the verification of (3.11) is by showing that: the matrix $Z$ defined by $(Z)_{i, j}=\left((\widetilde{N})^{l}\right)_{0, j}, i, j \geqq 0$, is lower triangular with 1 's on the diagonal, and satisfies Eq. (3.9). (Then $Z=X$, by the uniqueness assertion proved above.)

The fact that $Z$ is lower triangular is immediate: the diagonal height (defined as in 1.1.B) of $\check{N}$ is obviously $d h(\check{N})=1$, which implies $d h\left((\check{N})^{i}\right) \leqq i$ for $i \geqq 0$, and has as consequence that $\left((\breve{N})^{i}\right)_{0, j}=0$ for $j>i$. Moreover, the same kind of argument shows that for every $i \geqq 2$, the sum:

$$
\left((\check{N})^{i}\right)_{0, i}=\sum_{l_{1}, \ldots, l_{i-1}=0}^{\infty}(\check{N})_{0, l_{1}}(\check{N})_{l_{1}, l_{2}} \cdots(\check{N})_{l_{l-1}, i}
$$


reduces in fact to the single term $(\check{N})_{0,1}(\check{N})_{1,2} \cdots(\check{N})_{i-1, i}=(N)_{1,1}(N)_{2,2} \cdots(N)_{i, i}=$ 1. Since it is also clear that $\left((\check{N})^{0}\right)_{0,0}=\left((\check{N})^{1}\right)_{0,1}=1$, it follows that $Z$ has indeed 1 's on the diagonal.

Finally, we verify (3.9). Since both $Z$ and $(1 \oplus Z) N$ have the first line equal to $(1,0,0, \ldots)$, it suffices to check $((1 \oplus Z) N)_{i, j}=(Z)_{i, j}$ for $i \geqq 1$. We have:

$$
((1 \oplus Z) N)_{l, j}=\sum_{k=1}^{\infty}(1 \oplus Z)_{i, k}(N)_{k, j}
$$

(the sum is from $k=1$ because $\left.(1 \oplus Z)_{i, 0}=0\right)$

$$
\begin{aligned}
& =\sum_{k=1}^{\infty}(Z)_{i-1, k-1}(\check{N})_{k-1, j}=\left(\text { by putting } l=k-1 \text { and replacing }(Z)_{l-1, l}\right) \\
& =\sum_{l=0}^{\infty}\left((\check{N})^{i-1}\right)_{0, l}(\check{N})_{l, j}=\left((\check{N})^{i-1} \cdot \check{N}\right)_{0, j}=(Z)_{i, j} \cdot \quad \text { QED }
\end{aligned}
$$

3.5. The proof of $(b) \Leftrightarrow(c)$ in Theorem 1.2. We remark that the matrices: $T_{\theta, q^{-}}$ defined by (1.11), and $\check{M}$-with $M$ defined by (1.13), are conjugated by a diagonal matrix. (To put things in the right order: $\check{M}$ is the unique matrix obtained from $T_{\theta, q}$ by conjugating with a diagonal matrix, such that we get 1 's on the first diagonal above the principal one.) Indeed, if $\Delta_{q}$ is the diagonal matrix considered in 3.1, $\left(\Delta_{q}\right)_{i, i}=\sqrt{[i]_{q} !}, i \geqq 0$, then the definition of $T_{\theta, q}$ combined with the formulae (3.1), (3.2) of 3.1 give us:

$$
\left(\Delta_{q}^{-1} T_{\theta, q} \Delta_{q}\right)_{i, j}= \begin{cases}\alpha_{i-j+1}, & \text { if } j \leqq i \\ {[j]_{q},} & \text { if } j=i+1 \\ 0, & \text { if } j>i+1\end{cases}
$$

By writing $\Delta_{q} T_{\theta, q} \Delta_{q}^{-1}$ as $\Delta_{q}^{2}\left(\Delta_{q}^{-1} T_{\theta, q} \Delta_{q}\right) \Delta_{q}^{-2}$ and using (3.14) it is then immediate that

$$
\Delta_{q} T_{\theta, q} \Delta_{q}^{-1}=\check{M} .
$$

Now, if $\Gamma$ is the unique solution of Eq. (1.14), then by the previous lemma we have, for every $n \geqq 0$ :

$$
(\Gamma)_{n, 0}=\left((\check{M})^{n}\right)_{0,0} \stackrel{(3.15)}{=}\left(\Delta_{q} T_{\theta, q}^{n} \Delta_{q}^{-1}\right)_{0,0}=\left(T_{\theta, q}^{n}\right)_{0,0},
$$

and the latter quantity was the definition taken for $\mu\left(X^{n}\right)$ at the beginning of this section. QED

\section{Remarks on the Interpolated Convolution Laws $\uplus_{q}$}

4.1. Definition. Let $q \in[0,1]$ be a parameter. We denote by $\uplus_{q}$ the operation on the space of distributions $\Sigma$ of (1.1), which is linearized by the $R_{q}$-transform $R_{q}: \Sigma \rightarrow \Theta$; i.e.,

$$
\mu_{1} \boxplus_{q} \mu_{2}=R_{q}^{-1}\left(R_{q}\left(\mu_{1}\right)+R_{q}\left(\mu_{2}\right)\right), \mu_{1}, \mu_{2} \in \sum .
$$

It is clear from the definition that $\left(\Sigma, \Theta_{q}\right)$ is an Abelian group, for every $0 \leqq$ $q \leqq 1$. From the considerations in Remarks 1.4, 1.5, it follows that $\uplus_{0}=\boxplus$, the 
free convolution of Voiculescu $([16,17])$, while $\bigoplus_{1}$ is the usual convolution of distributions.

This way of introducing the interpolated convolution (via the linearizing transform) has at least one immediate advantage; namely, that we get from the very beginning an algebraic version of what is usually called "the method of characteristic functions" (see, e.g. [4], Sect. XV.5). Thus, for instance, it is very easy to prove an interpolated version of the central limit theorem. Since our approach consisted in working with distributions (rather than with random variables), we consider the formulation of the central limit theorem in this language; that is, we use the $q$-convolution $\bigoplus_{q}$ of distributions as a counterpart for a notion of "addition of $q$-independent random variables." Also, the operation of multiplying a random variable by a constant $r>0$ finds its analogue in the one of composing a distribution with the dilation $D_{r}: \mathbf{C}\langle X\rangle \rightarrow \mathbf{C}\langle X\rangle\left(D_{r}=\right.$ the unique homomorphism such that $\left.D_{r}(X)=r X\right)$. Thus:

4.2. Central Limit Theorem. Let $q \in[0,1]$ be a parameter, and let $\left(\mu_{k}\right)_{k=1}^{\infty}$ be a sequence of distributions such that:

(i) $\mu_{k}(X)=0$ for every $k \geqq 1$;

(ii) $\lim _{k \rightarrow \infty} \frac{1}{k} \sum_{j=1}^{k} \mu_{j}\left(X^{2}\right)=1$;

(iii) $\sup _{k}\left|\mu_{k}\left(X^{n}\right)\right|<\infty$ for every $n \geqq 2$.

Then the sequence $\left(\left(\mu_{1} \bigoplus_{q} \cdots \bigoplus_{q} \mu_{n}\right) \circ D_{1 / \sqrt{n}}\right)_{n=1}^{\infty}$ converges weakly to the distribution $R_{q}^{-1}\left(z^{2}\right)$.

The sense of the words "converges weakly" in Theorem 4.2 is the one of convergence of moments of all orders. The " $q$-Gaussian" distribution $R_{q}^{-1}\left(z^{2}\right)$, playing the role of central limit, is the same which appeared in the considerations in 1.6 above. We mention that interpolations of the central limit theorem, with the same central limit laws, have been previously considered in [2], Sect. 4 (version equivalent to 4.2 above, but in language of random variables) and also in [12], in a different context.

The proof of Theorem 4.2 comes to repeating word by word the argument

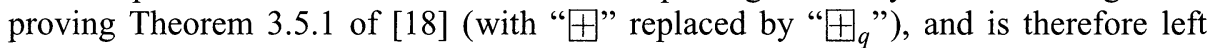
to the reader.

Now, of course, both usual and free convolution were originally defined in terms of a "product" which takes values outside $\Sigma$ (the tensor and the free product, respectively - see Eqs. (1.2), (1.3) above). It appears hence as a natural question whether one can define an operation with distributions, $\star_{q}, 0 \leqq q \leqq 1$, which interpolates between the free and the tensor product, and such that a formula of the type (1.2), (1.3) holds for every $q \in[0,1]$.

It is reasonable to ask that, for $q \in[0,1]$ and $\mu_{1}, \mu_{2} \in \Sigma$, the " $q$-free product" $\mu_{1} \star_{q} \mu_{2}$ is a linear functional on an algebra of polynomials in two indeterminates. Actually, we can always consider $\mu_{1} \star_{q} \mu_{2}$ on the algebra $\mathbf{C}\left\langle X_{1}, X_{2}\right\rangle$ of noncommutative polynomials in $X_{1}$ and $X_{2}$, by reasons of universality (any algebra of polynomials in two indeterminates is a quotient of $\mathbf{C}\left\langle X_{1}, X_{2}\right\rangle$, hence linear functionals on it can be pulled back to $\left.\mathbf{C}\left\langle X_{1}, X_{2}\right\rangle\right)$. In particular, for $q=1, \mu_{1} \star_{1} \mu_{2}$ : $\mathbf{C}\left\langle X_{1}, X_{2}\right\rangle \rightarrow \mathbf{C}$ will be the composition $\mathbf{C}\left\langle X_{1}, X_{2}\right\rangle \rightarrow \mathbf{C}\left\langle X_{1}\right\rangle \otimes \mathbf{C}\left\langle X_{2}\right\rangle \stackrel{\mu_{1} \otimes \mu_{2}}{\longrightarrow} \mathbf{C}$, or in other words will be described by

$$
\left(\mu_{1} \star_{1} \mu_{2}\right)\left(X_{i_{1}} \cdots X_{i_{n}}\right)=\mu_{1}\left(X^{h}\right) \mu_{2}\left(X^{k}\right), n \geqq 1, i_{1}, \ldots, i_{n} \in\{1,2\},
$$


where in (4.2) $h$ is the number of 1's and $k=n-h$ is the number of 2's appearing among $i_{1}, \ldots, i_{n}$. (In this context, the fact that the tensor product is really a functional on $\mathbf{C}\left\langle X_{2}\right\rangle \otimes \mathbf{C}\left\langle X_{2}\right\rangle$ is stated as " $\mu_{1} \star_{1} \mu_{2}$ can be factored through $\mathbf{C}\left\langle X_{1}\right\rangle \otimes \mathbf{C}\left\langle X_{2}\right\rangle$," and reflects the presence of some "additional symmetry," particular to the case $q=1$.

Hence, in short, the problem is that we have the formula

$$
\left(\mu_{1} \boxplus_{q} \mu_{2}\right)\left(X^{n}\right)=\left(\mu_{1} \star_{q} \mu_{2}\right)\left(\left(X_{1}+X_{2}\right)^{n}\right), n \geqq 0, \mu_{1}, \mu_{2} \in \sum, q=0,1 ，
$$

and we would like to extend it to the case of arbitrary $q \in[0,1]$. The key idea in order to do this appears to be (once again) to apply an adequate "transform," which makes the formuale defining $\mu_{1} \star_{0} \mu_{2}$ and $\mu_{1} \star_{1} \mu_{2}$ look very similar. More precisely, we will define a two-dimensional analogue $R_{I, q}$ of the $R_{q}$-transform, $0 \leqq q \leqq 1$, and it will turn out that for both $q=0$ and $q=1$ we have the very simple formula:

$$
\left(R_{I I, q}\left(\mu_{1} \star_{q} \mu_{2}\right)\right)\left(z_{1}, z_{2}\right)=\left(R_{q}\left(\mu_{1}\right)\right)\left(z_{1}\right)+\left(R_{q}\left(\mu_{2}\right)\right)\left(z_{2}\right) .
$$

Then (4.4) will be used to define $\mu_{1} \star_{q} \mu_{2}$ for arbitrary $q \in[0,1]$. The details of this are presented in the following

4.3. Definition. $1^{o}$ We denote by $\Sigma_{I I}$ the space of linear functionals $\mu$ : $\mathbf{C}\left\langle X_{1}, X_{2}\right\rangle \rightarrow \mathbf{C}$ such that $\mu(1)=1$; and by $\Theta_{I I}$ the space of formal power series in two non-commuting variables, and with vanishing constant coefficient. An element $\theta \in \Theta_{I I}$ is thus of the form:

$$
\theta\left(z_{1}, z_{2}\right)=\sum_{n \geqq 1} \sum_{i_{1}, \ldots, i_{n} \in\{1,2\}} \beta_{l_{1}, \ldots, i_{n}} z_{i_{1}} \cdots z_{i_{n}}
$$

with $\left(\beta_{i_{1}, \ldots, l_{n}}\right)_{n \geqq 1 ; i_{1}, \ldots, i_{n} \in\{1,2\}}$ complex coefficients.

For $\theta \in \Theta_{I I}, n \geqq 1$ and $i_{1}, \ldots, i_{n} \in\{1,2\}$, we will denote by $\langle\theta\rangle_{i_{1}, \ldots, i_{n}}$ the coefficient of $z_{i_{1}} \cdots z_{l_{n}}$ in $\theta$. Also, the following notation concerning multi-indices will be useful: If $i_{1}, \ldots, i_{n} \in\{1,2\}$ and $\emptyset \neq B \subseteq\{1, \ldots, n\}$ is a subset with, say, $m$ elements, then $\left(i_{1}, \ldots, i_{n}\right) \mid B$ will denote the $m$-tuple obtained by deleting from $i_{1}, \ldots, i_{n}$ the $i_{k}$ 's with $k \notin B$. (For example, $(1,2,2,1,2,1) \mid\{2,4,5,6\}=(2,1,2,1)$.)

$2^{\circ}$ Let $q \in[0,1]$ be a parameter. We define $R_{I I, q}^{-1}: \Theta_{I I} \rightarrow \Sigma_{I I}$ by associating to $\theta \in \Theta_{I I}$ the functional $\mu \in \Sigma_{I I}$ determined by:

$$
\mu\left(X_{i_{1}} \cdots X_{i_{n}}\right)=\sum_{\substack{\pi \in \mathscr{P}(\{1, \ldots, n\}) \\ \pi=\left\{B_{1}, \ldots, B_{m}\right\}}} q^{c_{o}(\pi)} \prod_{k=1}^{m}\left[\left|B_{k}\right|-1\right]_{q} !\langle\theta\rangle_{\left(i_{1}, \ldots, i_{n}\right) \mid B_{k}} .
$$

Then $R_{I I, q}^{-1}$ is easily seen to be a bijection from $\Theta_{I I}$ onto $\Sigma_{I I}$, and we define $R_{I I, q}: \Sigma_{I I} \rightarrow \Theta_{I I}$ to be $\left(R_{I I, q}^{-1}\right)^{-1}$.

$3^{o}$ Let $q \in[0,1]$ be a parameter. For every $\mu_{1}, \mu_{2}$ in the space of distributions $\Sigma$ of (1.1), we define their q-free product $\mu_{1} \star_{q} \mu_{2}$ to be the unique linear functional in $\Sigma_{I I}$ which satisfies Eq. (4.4).

For the fact that $\star_{0}$ defined by $4.3 .3^{\circ}$ coincides with the free product of distributions, see Theorem 3.3 of [9] (in a slightly different context, this phenomenon was first put into evidence in Sect. 4. of [13]). The fact that $\star_{1}$ defined by $4.3 .3^{\circ}$ 
is the same as the one appearing in Eq. (4.2), comes to an easy calculation made in the following

4.4. Lemma. Let $\mu_{1}, \mu_{2}$ be in $\Sigma$, and let $\theta \in \Theta_{I I}$ be the formal power series defined by $\theta\left(z_{1}, z_{2}\right) \equiv\left(R_{1}\left(\mu_{1}\right)\right)\left(z_{1}\right)+\left(R_{1}\left(\mu_{2}\right)\right)\left(z_{2}\right)$. Then for every $n \geqq 1$ and $i_{1}, \ldots, i_{n} \in$ $\{1,2\}$, we have that $\left(R_{I I, 1}^{-1}(\theta)\right)\left(X_{l_{1}} \cdots X_{l_{n}}\right)$ coincides with the right-hand side of (4.2).

Proof. Let us fix $n \geqq 1$ and $i_{1}, \ldots, i_{n} \in\{1,2\}$, and make the notation $C_{1}=\{1 \leqq$ $\left.m \leqq n \mid i_{m}=1\right\}, C_{2}=\left\{1 \leqq m \leqq n \mid i_{m}=2\right\}$. We have, by the definition of $R_{I I, 1}^{-1}$ :

$$
\left(R_{I I, 1}^{-1}(\theta)\right)\left(X_{l_{1}} \cdots X_{i_{n}}\right)=\sum_{\substack{\pi \in \mathcal{P}(\{1, \ldots, n\}) \\ \pi=\left\{B_{1}, \ldots, B_{m}\right\}}} \prod_{k=1}^{m}\left(\left|B_{k}\right|-1\right) !\langle\theta\rangle_{\left(l_{1}, \ldots, i_{n}\right) \mid B_{k}}
$$

in view of the particular form of $\theta$, this can be continued with:

$$
\sum_{\substack{\pi_{1} \in \mathscr{P}\left(C_{1}\right), \pi_{2} \in \mathscr{P}\left(C_{2}\right) \\ \pi_{1}=\left\{B_{1}^{(1)}, \ldots, B_{m_{1}}^{(1)}\right\} \\ \pi_{2}=\left\{B_{1}^{(2)}, \ldots, B_{m_{2}}^{(2)}\right\}}}^{\prod_{k=1}^{m_{1}}\left(\left(\left|B_{k}^{(1)}\right|-1\right) ! \alpha_{\left|B_{k}^{(1)}\right|}^{(1)}\right) \prod_{k=1}^{m_{2}}\left(\left(\left|B_{k}^{(2)}\right|-1\right) ! \alpha_{\left|B_{k}^{(2)}\right|}^{(2)}\right),}
$$

where $\left(\alpha_{l}^{(1)}\right)_{l=1}^{\infty}$ and $\left(\alpha_{l}^{(2)}\right)_{l=1}^{\infty}$ are the coefficients of the formal power series $R_{1}\left(\mu_{1}\right)$ and $R_{1}\left(\mu_{2}\right)$, respectively. But clearly, the sum (4.6) decomposes as a product of two factors, which equals (by characterization (a) of Theorem 1.2) $R_{1}^{-1}\left(R_{1}\left(\mu_{1}\right)\right)\left(X^{\left|C_{1}\right|}\right) \cdot R_{1}^{-1}\left(R_{1}\left(\mu_{2}\right)\right)\left(X^{\left|C_{2}\right|}\right)=\mu_{1}\left(X^{\left|C_{1}\right|}\right) \mu_{2}\left(X^{\left|C_{2}\right|}\right)$. QED

Hence the operations $\star_{q}, 0 \leqq q \leqq 1$, defined by $4.3 .3^{\circ}$, really interpolate between the free product and (the pull-back of) the tensor product, and it only remains to verify that

4.5. Theorem. Relation (4.3) holds for every parameter $q \in[0,1]$ and every $\mu_{1}, \mu_{2} \in \Sigma$.

Proof. Let us fix $q \in[0,1]$ and $\mu_{1}, \mu_{2} \in \Sigma$, and let us denote the $R_{q}$-transforms of $\mu_{1}$ and $\mu_{2}$ by $\theta_{1}(z)=\sum_{n=1}^{\infty} \alpha_{n}^{(1)} z^{n}$ and $\theta_{2}(z)=\sum_{n=1}^{\infty} \alpha_{n}^{(2)} z^{n}$, respectively. Then the $R_{I I, q}$-transform of $\mu_{1} \star_{q} \mu_{2}$ is (by Definition $4.3 .3^{\circ}$ ) the formal power series $\theta \in \Theta_{I I}$ defined by $\theta\left(z_{1}, z_{2}\right) \equiv \theta_{1}\left(z_{1}\right)+\theta_{2}\left(z_{2}\right)$.

Let us also fix $n \geqq 2$. We have

$$
\begin{gathered}
\left(\mu_{1} \star_{q} \mu_{2}\right)\left(\left(X_{1}+X_{2}\right)^{n}\right)=\sum_{\left(i_{1}, \ldots, i_{n}\right) \in\{1,2\}^{n}}\left(\mu_{1} \star_{q} \mu_{2}\right)\left(X_{l_{1}} \cdots X_{i_{n}}\right) \\
=\sum_{\left(l_{1}, \ldots, i_{n}\right) \in\{1,2\}^{n}} \sum_{\substack{\pi \in \mathscr{P}(\{1, \ldots, n\}) \\
\pi=\left\{B_{1}, \ldots, B_{m}\right\}}} q^{c_{o}(\pi)} \prod_{k=1}^{m}\left[\left|B_{k}\right|-1\right]_{q} !\langle\theta\rangle_{\left(l_{1}, \ldots, l_{n}\right) \mid B_{k}}
\end{gathered}
$$

(where we used that $\mu_{1} \star_{q} \mu_{2}=R_{I I, q}^{-1}(\theta)$, and formula (4.5)).

Now, given an $n$-tuple $\left(i_{1}, \ldots, i_{n}\right) \in\{1,2\}^{n}$ and a partition $\pi \in \mathscr{P}(\{1, \ldots, n\})$, let us write " $\left(i_{1}, \ldots, i_{n}\right) \prec \pi$ " if $i_{h}=i_{l}$ whenever $1 \leqq h \leqq l \leqq n$ are in the same class of $\pi$. Remark that in the double sum (4.7), the term corresponding to $\left(i_{1}, \ldots, i_{n}\right) \in\{1,2\}^{n}$ and $\pi=\left\{B_{1}, \ldots, B_{m}\right\} \in \mathscr{P}(\{1, \ldots, n\})$ can be non-zero only if 
$\left(i_{1}, \ldots, i_{n}\right) \prec \pi$. Indeed, in the opposite case we can find a class $B_{k}$ of $\pi, 1 \leqq k \leqq m$, such that $\left(i_{1}, \ldots, i_{n}\right) \mid B_{k}$ contains both 1's and 2's; then $\langle\theta\rangle_{\left(i_{1}, \ldots, i_{n}\right) \mid B_{k}}=0$, forcing the corresponding term of the sum (4.7) to vanish.

Taking into account the remark of the preceding paragraph, and changing the order of summation in (4.7), we obtain:

$$
\left(\mu_{1} \star_{q} \mu_{2}\right)\left(\left(X_{1}+X_{2}\right)^{n}\right)=\sum_{\substack{\pi \in \mathscr{P}(\{1, \ldots, n\}) \\ \pi=\left\{B_{1}, \ldots, B_{m}\right\}}} q^{c_{o}(\pi)} \prod_{k=1}^{m}\left[\left|B_{k}\right|-1\right]_{q} ! \sum_{\left(i_{1}, \ldots, i_{n}\right) \prec \pi} \prod_{k=1}^{m}\langle\theta\rangle_{\left(i_{1}, \ldots, i_{n}\right) \mid B_{k}} .
$$

Let us next take a partition $\pi=\left\{B_{1}, \ldots, B_{m}\right\} \in \mathscr{P}(\{1, \ldots, n\})$, and concentrate for this $\pi$ on the second sum on the right-hand side of (4.8). The index set of this sum consists of those $2^{m}$ sequences $\left(i_{1}, \ldots, i_{n}\right) \in\{1,2\}^{n}$ which, viewed as functions from $\{1, \ldots, n\}$ to $\{1,2\}$, are constant on each class of $\pi$. Moreover, it is clear (from $\left.\theta\left(z_{1}, z_{2}\right) \equiv \theta_{1}\left(z_{1}\right)+\theta_{2}\left(z_{2}\right)\right)$ that for every such sequence $\left(i_{1}, \ldots, i_{n}\right)$ and every $1 \leqq k \leqq m$, we have

$$
\langle\theta\rangle_{\left(i_{1}, \ldots, l_{n}\right) \mid B_{k}}= \begin{cases}\alpha_{\left|B_{k}\right|}^{(1)}, & \text { if }\left(i_{1}, \ldots, i_{n}\right) \text { is constantly } 1 \text { on } B_{k} \\ \alpha_{\left|B_{k}\right|}^{(2)}, & \text { if }\left(i_{1}, \ldots, i_{n}\right) \text { is constantly } 2 \text { on } B_{k}\end{cases}
$$

(where recall that $\left(\alpha_{l}^{(1)}\right)_{l=1}^{\infty}$ and $\left(\alpha_{l}^{(2)}\right)_{l=1}^{\infty}$ are the coefficients of $\theta_{1}$ and $\theta_{2}$, respectively). In this way we see that:

$$
\begin{aligned}
\sum_{\left(i_{1}, \ldots, i_{n}\right) \prec \pi} \prod_{k=1}^{m}\langle\theta\rangle_{\left(i_{1}, \ldots, i_{n}\right) \mid B_{k}} & =\sum_{\left(j_{1}, \ldots, j m\right) \in\{1,2\}^{m}} \alpha_{\left|B_{1}\right|}^{\left(j_{1}\right)} \cdots \alpha_{\left|B_{m}\right|}^{\left(j_{m}\right)} \\
& =\prod_{k=1}^{m}\left(\alpha_{\left|B_{k}\right|}^{(1)}+\alpha_{\left|B_{k}\right|}^{(2)}\right) .
\end{aligned}
$$

Hence, returning to $(4.8)$, we conclude that it can be continued with:

$$
\begin{aligned}
\sum_{\substack{\pi \in \mathscr{P}\{\{1, \ldots, n\}) \\
\pi=\left\{B_{1}, \ldots, B_{m}\right\}}} q^{c_{o}(\pi)} \prod_{k=1}^{m}\left[\left|B_{k}\right|-1\right]_{q} !\left(\alpha_{\left|B_{k}\right|}^{(1)}+\alpha_{\left|B_{k}\right|}^{(2)}\right) \\
=\left[R_{q}^{-1}\left(\theta_{1}+\theta_{2}\right)\right]\left(X^{n}\right)(\text { by (a) of Theorem 1.2) } \\
=\left(\mu_{1} \bigoplus_{q} \mu_{2}\right)\left(X^{n}\right)\left(\text { by the definition of } \bigoplus_{q}\right) . \quad \text { QED }
\end{aligned}
$$

Acknowledgements. The results in this paper are part of the author's Ph.D. Thesis at the University of California at Berkeley. I would like to express my gratitude to my advisor, Dan Voiculescu, for his constant encouragements and support, and for his generosity in providing me with an abundance of exciting (and at the same time workable) problems.

I would also like to express my thanks to Ken Dykema for useful discussions at an early stage of this work.

\section{References}

1. Askey, R., Ismail, M.: Recurrence relations, continued fractions and orthogonal polynomials. Mem. of the Am. Math. Soc. 49, No. 300, (1984)

2. Bożejko, M.: A $q$-deformed probability, Nelson's inequality and central limit theorems. In: Non-linear fields: classical, random, semiclassical, P. Garbaczewski, Z. Popowicz (eds.) 1991, pp. $312-335$ 
3. Bożejko, M., Speicher, R.: An example of a generalized Brownian motion. Part I in Commun. Math. Phys. 137, 519-531, 1991; Part II in Quantum probability and related topics VII (L. Accardi, editor), 1990, pp. 67-77

4. Feller, W.: An introduction to probability theory and its applications. Volume 2, New York: Wiley, 1966

5. Flajolet, Ph.: Combinatorial aspects of continued fractions. Discrete Math. 32, 125-161 (1980)

6. Gessel, I.M.: A $q$-analog of the exponential formula. Discrete Math. 40, 69-80 (1982)

7. Ismail, M.E.H., Stanton, D., Viennot, G.: The combinatorics of $q$-Hermite polynomials and the Askey-Wilson integral. Europ. J. Combinatorics 8, 379-392 (1987)

8. Kreweras, G.: Sur les partitions non-croisées d'un cycle. Discrete Math. 1, 333-350 (1972)

9. Nica, A.: $R$-transforms of free joint distributions, and non-crossing partitions. Preprint

10. Nica, A.: Ph.D. Thesis, University of California at Berkeley, May 1994

11. Shiryayev, A.N.: Probability. Berlin, Heidelberg, New York: Springer 1984

12. Speicher, R.: A non-commutative central limit theorem. Math Zeitschrift 209, 55-66 (1992)

13. Speicher, R.: Multiplicative functions on the lattice of non-crossing partitions and free convolution. Math. Annalen 298, 611-628 (1994)

14. Stanley, R.P.: Enumerative combinatorics. Volume I, Wadsworth \& Brooks / Cole Mathematics Series, 1986

15. Touchard, J.: Sur un problème de configurations et sur les fractions continues. Canadian J. Math. 4, 2-25 (1952)

16. Voiculescu, D.: Symmetries of some reduced free product $C^{*}$-algebras. In: Operator algebras and their connection with topology and ergodic theory, H. Araki, C.C. Moore, S. Stratila, D. Voiculescu (eds.) Springer Lecture Notes in mathematics, Volume 1132, Berlin Heidelberg, New York: Springer, 456-588 (1985)

17. Voiculescu, D.: Addition of certain non-commuting random variables. J. Funct. Anal. 66, 323-346 (1986)

18. Voiculescu, D.V., Dykema, K.J., Nica, A.: Free random variables. CRM Monograph Series, Volume 1, Providence RI: AMS, 1993

19. Wall, H.S.: Analytic theory of continued fractions, Amsterdam: van Nostrand, 1948

20. Woronowicz, S.L.: Twisted $S U(2)$ group. An example of a non-commutative differential calculus. Publ. RIMS, Kyoto Univ. 23, 117-181 (1987)

Communicated by A. Jaffe 
